\title{
Correlation between anterior chamber characteristics and laser flare photometry immediately after femtosecond laser treatment before phacoemulsification
}

${ }^{1}$ Department of Ophthalmology, CharitéUniversitätsmedizin Berlin, Berlin, Germany

${ }^{2}$ Augenklinik am Wittenbergplatz, Berlin, Germany

Correspondence:

M Pahlitzsch, Department of Ophthalmology, CharitéUniversitätsmedizin Berlin, Augustenburger Platz 1 , 13353 Berlin, Germany Tel: +004930450654325; Fax: +00493045065476 E-mail: milena.pahlitzsch@ charite.de

${ }^{3}$ These authors contributed equally to this work.

Received: 12 October 2015 Accepted in revised form: 15 April 2016 Published online: 27 May 2016

\begin{abstract}
Purpose To assess the anterior chamber (AC) characteristics and its correlation to laser flare photometry immediately after femtosecond laser-assisted capsulotomy and photodisruption.

Patients and methods The study included 97 cataract eyes ( $n=97$, mean age 68.6 years) undergoing femtosecond laser-assisted cataract surgery (FLACS). Three cohorts were analysed relating to the flare photometry directly post femtosecond laser treatment (flare $<100 n=28,69.6 \pm 7$ years; flare 100-249 $n=47,67.7 \pm 8$ years; flare $>249$ photon counts per ms cohort $n=22,68.5 \pm 10$ years). Flare photometry (KOWA FM-700), corneal topography (Oculus Pentacam, Germany: AC depth, volume, angle, pachymetry), axial length, pupil diameter, and endothelial cells were assessed before FLACS, immediately after femtosecond laser treatment and 1 day postoperative (LenSx Alcon, USA). Statistical data were analysed by SPSS v19.0, Inc. Results The AC depth, AC volume, AC angle, central and thinnest corneal thickness showed a significant difference between flare $<100$ vs flare 100-249 $10 \mathrm{~min}$ post femtosecond laser procedure $(P=0.002$, $P=0.023, P=0.007, P=0.003, P=0.011$, respectively). The $\mathrm{AC}$ depth, $\mathrm{AC}$ volume, and AC angle were significantly larger $(P=0.001$, $P=0.007, P=0.003$, respectively) in the flare $<100$ vs flare $>249$ cohort 10 min post femtosecond laser treatment.

Conclusions A flat AC, low AC volume, and a narrow $\mathrm{AC}$ angle were parameters associated with higher intraocular inflammation. These criteria could be used for patient selection in
\end{abstract}

M Pahlitzsch ${ }^{1}$, N Torun ${ }^{1}$, ML Pahlitzsch ${ }^{1}$, MKJ Klamann', J Gonnermann ${ }^{1}$, E Bertelmann ${ }^{1,3}$ and T Pahlitzsch ${ }^{2,3}$

FLACS to reduce postoperative intraocular inflammation.

Eye (2016) 30, 1110-1117; doi:10.1038/eye.2016.110; published online 27 May 2016

\section{Introduction}

Femtosecond laser-assisted cataract surgery (FLACS) is the latest revolution in the long history of cataract surgery. In comparative studies, FLACS produced more precise and reproducible capsulotomies than manual procedures. ${ }^{1-4}$ In addition, improved intraocular lens placement, capsule overlap, circularity of capsulotomy and centration of the intraocular lens were compared with manual procedures. ${ }^{1-4}$ The corneal incision by the femtosecond laser was stable and did not significantly change the higher order aberrations. ${ }^{5}$ Side effects including miosis, ocular inflammation, and alternated anterior chamber $(\mathrm{AC})$ characteristics were experienced in few patients. ${ }^{1-4,6}$ Another study demonstrated the impact of different pulse energy levels on the tissue outcome after capsulorhexis performance. ${ }^{6}$ A soft contact lens interface with a pulse energy of $5 \mu \mathrm{J}$ resulted in fewer tags and bridges, smoother edges, and a more regular and thinner demarcation line on specimen edges with the femtosecond laserperformed capsulotomies versus a rigid and curved $15 \mu \mathrm{J}$ interface application using light and scanning electron microscopy. ${ }^{6}$ Thus, energy levels interacted differently with tissue and influenced the ocular inflammation reaction.

Cells in the AC and flare were two parameters that were used to detect a blood-aqueous border break. ${ }^{7}$ Flare was graded by the intensity of light 
reflected by those proteins as it passed through aqueous humour. ${ }^{7}$ Cells were still calculated by the slit lamp examination. ${ }^{8}$ In the 1980s, the KOWA Company (Tokyo, Japan) developed techniques to quantify the amount of proteins in the AC noninvasively using a scanning laser. The laser flare photometry provided an accurate, reproducible and non-invasive assessment of aqueous flare that could prove valuable in research and clinical decisions. ${ }^{10}$

The aim of this study was to assess the AC characteristics and its correlation to the laser flare photometry immediately after femtosecond laser-assisted capsulotomy and photodisruption; the idea was to identify structural changes that were likely to lead to corneal reactions and increases in intraocular inflammation in some patients, while most patients did not suffer from side effects.

\section{Materials and methods}

Data from 97 normal cataract patients (97 eyes, mean age 68.6 years) were included in this prospective pilot study from June 2014 to January 2015 according to the Declaration of Helsinki Principle.

For each patient an ophthalmologic examination with medical history, best-corrected visual acuity, slit lamp examination, and fundoscopy was performed. Furthermore, corneal topography (Oculus Pentacam, Oculus Inc., Wetzlar, Germany) and flare analysis (Laser flare photometry, FM-700 Kowa, Japan) were carried out. The results of the flare photometry were split in three groups according to the flare values $10 \mathrm{~min}$ post femtosecond laser treatment; flare $<100$ photon counts per ms cohort ( $n=28,69.6 \pm 7.6$ years), flare $100-249$ photon counts per ms cohort ( $n=47,67.7 \pm 8.5$ years) and flare $>249$ photon counts per ms cohort $(n=22$, $68.5 \pm 10.0$ years). Parameters of different AC characteristics were analysed between the three flare cohorts. The borders of the flare photometry were chosen according to the results from Shah et al ${ }^{11}$ regarding in vitro and in vivo flare analysis in manual phacoemulsification.

The following $\mathrm{AC}$ characteristics were observed: $\mathrm{AC}$ depth, AC volume, AC angle, central corneal thickness and thinnest corneal thickness, axial length, white-towhite distance, pupil diameter, and number of endothelial cells. All data were conducted preoperatively and within maximum $20 \mathrm{~min}$ after femtosecond laser treatment before manual phacoemulsification and 1 day after surgery. The intraocular lens calculation was provided by the IOL Master (Zeiss, Meditec AG, Jena, Germany).

Inclusion criteria were a nuclear density score LOCS II, no other surgery $<3$ months and a systemic disease in a stable condition (diabetes Hba1c maximum $7 \%$ without any accompanying disease and hypertensive blood pressure in cataract patients). Exclusion criteria were LOCS I, III, or IV, uncontrolled systemic disease (diabetes, hypertensive blood pressure), autoimmune disease, uveitis, keratitis, hazy optic media, and a very shallow AC (limit of laser flare photometry).

\section{Surgery technique}

The femtosecond laser procedures were performed using the LenSx laser (Alcon Laboratories, Inc., Fort Worth, TX, USA) under topical anaesthesia with Conjucain EDO (oxybuprocainhydrochloride, $4.0 \mathrm{mg} / \mathrm{ml}$, Dr. Mann Pharma $\mathrm{GmbH}$, Berlin, Germany) by the same surgeon (TP). The procedure was performed in the operating suite. The LenSx laser is a $50 \mathrm{kHz}$ femtosecond infrared laser with a pulse width of 600-800 fs, a central laser wavelength of $1030 \mathrm{~nm}$ and maximum pulse energy of $15 \mu \mathrm{J}$. The laser pulse energy setting for performing the anterior capsulotomy was 4.8 and $8.9 \mu \mathrm{J}$ for the nucleus (offset up $200 \mu \mathrm{m}$, down $250 \mu \mathrm{m}$ ). The depth and coordinates of the capsulotomies were determined by the laser system's optical coherence tomography (OCT, Figure 1). The diameter of the capsulotomy was set to $5 \mathrm{~mm}$.

The interface was built as an extended suction skirt and an additional soft contact lens between the applanation lens and the cornea, which allowed a decrease of laser pulse energy while maintaining the ability to perform capsulotomies.

After docking, the cornea was applanated and suction was activated. Undocking was performed after laser treatment, and the interface with the contact lens was removed. After the measurements (maximum time period $20 \mathrm{~min}$ ), phacoemulsification was performed with the Alcon Infiniti Vision System. The corneal wound was created by blade so that the intraocular space was still enclosed during the examinations and thus minimised the infection risk as much as possible. Due to the soft interface and the low laser energy settings, no miosis was observed and thus none of the patients needed a pupil dilation between procedures. Postoperative treatment was comprised of a topical combination of steroids and antibiotics that were reduced over 4 weeks following the intervention. Before and during the examinations no topical or systemic medications were used.

\section{Flaremeter KOWA FM-700}

In principle, laser flare photometry uses a laser beam that scans a measurement window projected into the AC. Here, the KOWA FM-700 (Kowa Company, Ltd., ELECTRONICS AND OPTICS DIVISion, Tokyo, Japan) used a semiconductor diode laser $(640 \mathrm{~nm})$. The $\mathrm{He}-\mathrm{Ne}$ 


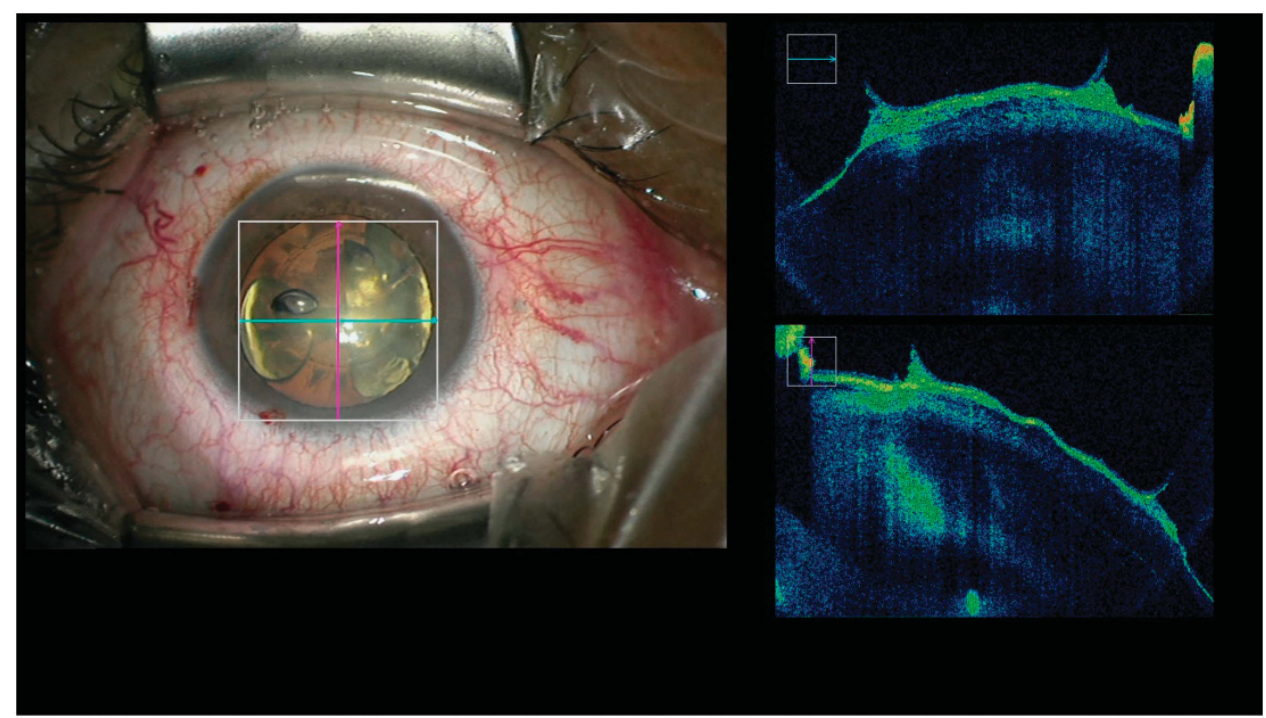

Figure 1 Clinical picture of the photodisruption and capsulotomy after femto laser procedure (left site); Edges of the capsulotomy shown on the intraoperative OCT after femtosecond laser photodisruption (right site).

beam had a power of $35 \mu \mathrm{W}$. The beam was projected into the $\mathrm{AC}$, and scattering of the beam within the sampling window was detected by the photomultiplier.

The measurement mode was the protein concentration setting. The laser beam was scanned vertically covering the sampling window. Scattering of this beam was measured when it passed through this window. Measurements were also taken when the beam passed above and below the sampling window to assess the background signal (BG1 and BG2). The flare value in photon counts/ms was calculated by subtracting the mean of the two background counts from the signal value.

The measurement range was 1-500 photon counts/ms. The slit width was $0-11 \mathrm{~mm}$ and was continuously available. $^{9,10}$

OCULUS Pentacam (Oculus, Inc.) images were obtained under pupil dilatation with $1 \%$ tropicamide. To reduce operator-dependent variables, Pentacam's automatic release mode was used. In this mode, the instrument automatically determined when correct focus and alignment with the corneal apex had been achieved and then performed a scan. In $<2 \mathrm{~s}$, the rotating camera captures up to 50 slit images of the anterior segment. ${ }^{12}$

The Pentacam Scheimpflug densitometric method using numerically graded blue light-scattering of the lens layers, was used to measure the nuclear density in the Lens Opacities Classification System (LOCS). ${ }^{12}$

Pupillometry was measured by the Colvard Pupillometer (OASIS Medical, Glendora, CA, USA). This precision instrument measures pupillary dilation in low levels of illumination. The Colvard Pupillometer is analogous to a direct ophthalmoscope. A lithium energy source operated the instrument.

\section{Statistical analysis}

We used SPSS to assess statistical data (SPSS version 19.0, SPSS, Inc., Armonk, NY, USA). Linear regression analysis and descriptive statistics (mean $\pm \mathrm{SD}$ ) were processed. Preoperative and postoperative values were compared with the paired Wilcoxon test. The Wilcoxon Test was used to compare two related samples or repeated measurements on a single sample. To test for general significant differences ANOVA was used for parametric variables and Kruskal-Wallis Test for non-parametric data. In addition, each of the flare cohorts vs the other two groups were separately analysed using the MannWhitney $U$-test as a post hoc analysis for numerical variables that were not normally distributed. For testing normality of our sample the Kolmogorov-Smirnov test was applied. A $P$-value of $<0.05$ indicated a statistically significant difference.

\section{Results}

Data of three cohorts were analysed; flare $<100$ photon counts per ms cohort ( $n=28,69.6 \pm 7.6$ years), flare 100 249 photon counts per ms cohort ( $n=47,67.7 \pm 8.5$ years) and flare $>249$ photon counts per ms cohort $(n=22$, $68.5 \pm 10.0$ years). A comparison of the AC and cornea characteristics in the three study cohorts were shown in Tables 1 and 2 . 
Table 1 Descriptive statistics (mean \pm standard deviation, minimum, maximum, $n=$ number) and $P$-value of different anterior chamber (AC) and cornea characteristics (AC depth, AC volume, AC angle, corneal thickness (CT), central corneal thickness (CCT), endothelial cell (CD)) in the complete study population according to three measuring times-preoperatively, 10 min post femtosecond laser treatment and 1 day follow up

\begin{tabular}{|c|c|c|c|}
\hline \multirow[t]{2}{*}{ Parameters } & \multicolumn{2}{|c|}{ Normal cataract } & \multirow[t]{2}{*}{ P-value pre- to postoperative comparison } \\
\hline & $M e a n \pm S D$ & Min-max, number & \\
\hline White to white & $11.98 \pm 0.36$ & $(11.00-12.70, n=97)$ & \\
\hline Axial length & $23.78 \pm 1.76$ & $(20.66-30.01, n=97)$ & \\
\hline Phaco time & $0.91 \pm 0.41$ & $(0.40-2.30, n=97)$ & \\
\hline AC depth pre-op & $2.59 \pm 0.41$ & $(1.59-3.29, n=97)$ & \\
\hline AC depth post-op & $2.45 \pm 0.44$ & $(1.44-3.19, n=97)$ & 0.001 \\
\hline AC depth post-op 1 day & $3.78 \pm 0.59$ & $(2.50-5.57, n=95)$ & 0.001 \\
\hline AC-Vol pre-op & $140.87 \pm 36.97$ & $(73.00-230, n=97)$ & \\
\hline AC-Vol post-op & $142.35 \pm 35.86$ & $(70.00-207.00, n=97)$ & 0.176 \\
\hline AC-Vol post-op day 1 & $147.02 \pm 36.10$ & $(69.00-231.00, n=97)$ & 0.376 \\
\hline AC angle pre-op & $28.64 \pm 8.22$ & $(6.20-56.20, n=97)$ & \\
\hline AC angle post-op & $31.81 \pm 10.28$ & $(6.10-56.70, n=97)$ & 0.014 \\
\hline AC angle post-op day 1 & $35.57 \pm 8.00$ & $(8.90-55.40, n=97)$ & $<0.001$ \\
\hline CCT pre-op & $556.76 \pm 29.19$ & $(474.00-625.00, n=97)$ & \\
\hline CCT post-op & $570.47 \pm 28.53$ & $(466.00-635.00, n=97)$ & 0.001 \\
\hline CCT post-op day 1 & $588.23 \pm 37.73$ & $(485.00-693.00, n=97)$ & $<0.001$ \\
\hline Thinnest CT pre-op & $553.31 \pm 30.06$ & $(473.00-623.00, n=97)$ & \\
\hline Thinnest CT post-op & $565.00 \pm 30.16$ & $(462.00-633.00, n=97)$ & 0.001 \\
\hline Thinnest CT post-op 1 day & $583.87 \pm 35.35$ & $(484.00-655.00, n=95)$ & $<0.001$ \\
\hline Flare pre-op & $12.31 \pm 6.77$ & $(4.00-35.70, n=97)$ & \\
\hline Flare post-op & $169.93 \pm 111.91$ & $(25.90-500.00, n=95)$ & 0.001 \\
\hline Flare post-op 1 day & $26.62 \pm 32.84$ & $(5.20-149.80, n=95)$ & 0.001 \\
\hline CD pre-op & $2555.07 \pm 271.72$ & $(1788.00-3121.00, n=97)$ & \\
\hline CD post-op & $2588.91 \pm 324.09$ & $(1732.00-3372.00, n=96)$ & 0.134 \\
\hline CD post-op 1 day & $2539.80 \pm 317.02$ & $(1575.00-3259.00, n=96)$ & 0.558 \\
\hline Pupil pre-op & $6.43 \pm 0.70$ & $(5.00-8.00, n=97)$ & \\
\hline Pupil post-op & $5.76 \pm 1.59$ & $(2.00-9.00, n=68)$ & 0.001 \\
\hline Pupil post-op 1 day & $6.19 \pm 1.26$ & $(3.00-8.00, n=95)$ & 0.051 \\
\hline
\end{tabular}

\section{Comparison of three different postoperative flare cohorts} due to corneal and AC characteristics

Flare values did not significantly differ between the three cohorts pre-operatively $(P>0.050)$; flare $<100$ cohort $11.68 \pm 8.56$ photon counts $/ \mathrm{ms}$, flare 100-249 cohort $11.95 \pm 8.88$ photon counts $/ \mathrm{ms}$ and flare $>249$ cohort $14.25 \pm 7.58$ photon counts $/ \mathrm{ms}$.

AC depth (Figure 2), AC volume and AC angle showed a significant difference between the flare $<100$ and flare 100-249 groups $10 \mathrm{~min}$ post femtosecond laser treatment $(P=0.002, P=0.023, P=0.007$ respectively, Table 2$)$. The central and thinnest corneal thickness showed a significant difference between the flare $<100$ and flare 100-249 groups $10 \mathrm{~min}$ post femtosecond laser $(P=0.003$, $P=0.011$, respectively, Figure 3 and Table 2).

The central and thinnest CT showed a significant difference between the flare 100-249 and flare $>249$ cohorts $10 \mathrm{~min}$ post femtosecond laser treatment $(P=0.046, P=0.023$, Table 2 and Figure 3$)$.

The AC depth $(P=0.001$, Figure 2$)$ and volume $(P=0.007)$ differed significantly between the flare $<100$ and flare $>249$ photon counts $/ \mathrm{ms}$ cohorts $10 \mathrm{~min}$ post femtosecond laser treatment. In addition, the AC angle differed significantly $10 \mathrm{~min}$ post femtosecond laser $(P=0.003)$ and 1 day follow up $(P=0.040)$ between the flare $<100$ and flare $>249$ cohorts. Accessorily, the number of endothelial cells showed a significant difference between the flare $<100$ and flare $>249$ photon counts $/ \mathrm{ms}$ cohorts at 1 day of follow up $(P=0.038$, Table 2$)$.

\section{Discussion}

FLACS is currently assessed by multiple studies concerning reduction of energy levels, endothelial loss and refractive outcome to identify patient cohorts, who would benefit from this newly developed procedure. ${ }^{1-4,6}$ Regarding safety features of the FLACS, studies have shown that the incidence of intraoperative complications such as suction breaks or anterior capsular tears decreased with experience and were $<2 \% .^{1-3}$ Macular thickness did not differ between FLACS and traditional phacoemulsification. ${ }^{1-3}$ Additionally, the Femtosecond laser-assisted anterior capsulotomy was a safe procedure for postoperative posterior capsule opacification rates due to better intraocular lens position. ${ }^{13}$ 


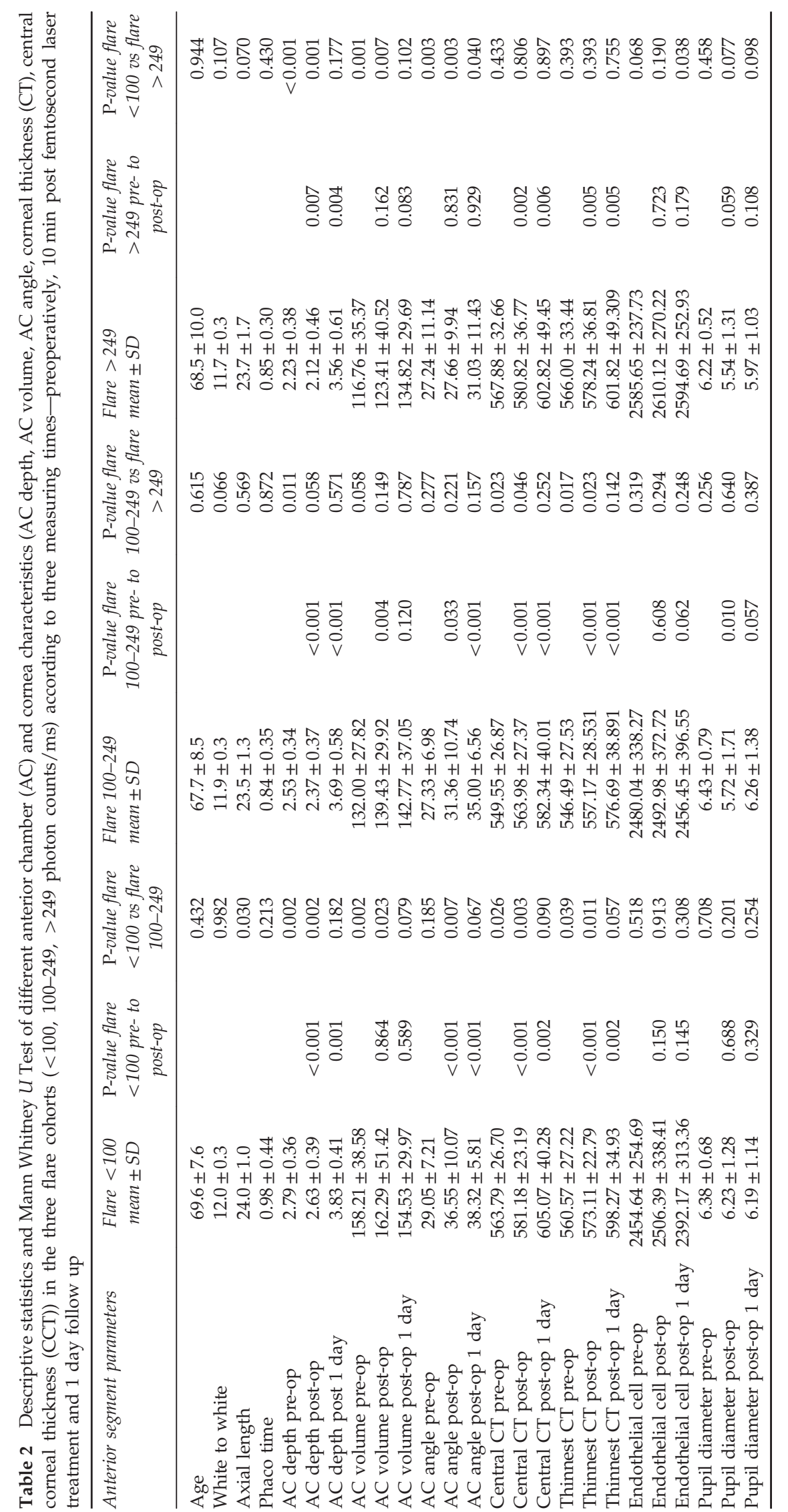




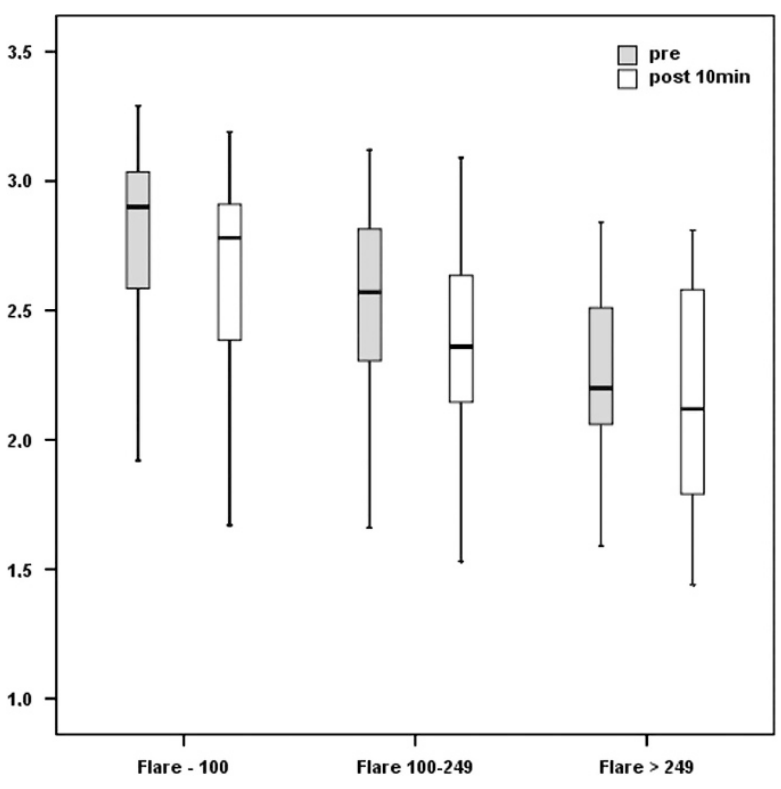

Figure 2 Boxplot analysis of the anterior chamber depth preoperatively and $10 \mathrm{~min}$ post femtosecond laser in the three flare analysis cohorts.

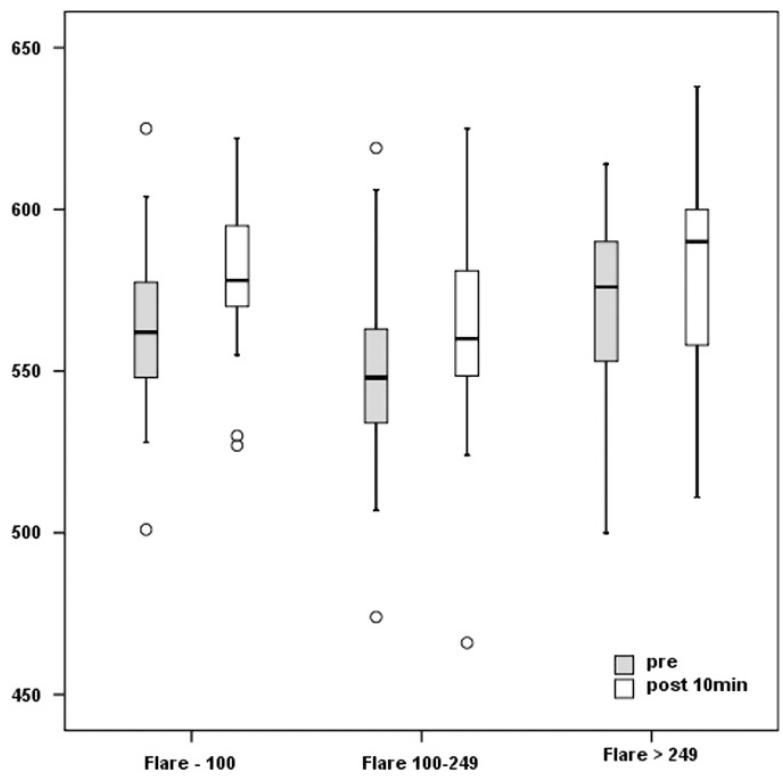

Figure 3 Boxplot analysis of the central cornea thickness preoperatively and $10 \mathrm{~min}$ post femtosecond laser in the three flare analysis cohorts.

Basic goal of this study was to analyse the AC characteristics altered by the procedure of femtosecond laserassisted capsulorhexis and photodisruption. Our findings may allow identifying variations in patient' outcomes.

Laser flare photometry is the golden standard to measure AC inflammation. ${ }^{10,14}$ Damage to the blood-aqueous barrier immediately after cataract surgery was related to the type of surgery, systemic diseases, incision size, bubble formation, and the mechanical trauma to the uveal tissues. ${ }^{2,15-18}$ Abell et $a l^{19}$ demonstrated that lower anterior segment inflammation was observed in FLACS versus manual surgery. Oshika et $a l^{16}$ have found an increase in flare photometry with age and corresponding higher preoperative flare value in older patients. ${ }^{14}$ In our study, no significant correlation of the factor age could be shown.

Shah et al ${ }^{11}$ summarised the flare analysis outcome: Flare counts after cataract surgery in normal eyes ranged from 30 to 250 immediately after surgery and between $96 \pm 65.3$ on the first postoperative day. Flare counts in acutely inflamed uveitic eyes usually were 50-400; the highest counts were 800-1000 in eyes with fibrinous exudates and hypopyon. ${ }^{11}$ Schauersberger et $a l^{20}$ assessed the long-term disorders of the blood-aqueous barrier after small-incision cataract surgery and found a mean difference of 2.12 photon counts $/ \mathrm{ms}$ between preoperative and 2 year results $(P<0.001)$. According to Shah et al ${ }^{11}$ findings, three different flare photometry cohorts were analysed considering the photometry measurements $10 \mathrm{~min}$ post femtosecond treatment. In line with the chosen time point of the cohort classification, we looked for significant differences in preoperative values of the AC parameters between the three flare cohorts to identify risk factors of increased intraocular inflammation in patients undergoing cataract surgery, without existing flare differences preoperatively (Table 2). AC depth, AC volume, AC angle, and corneal thickness showed significant differences between the three cohorts preoperatively, indicating a deeper AC in the flare $<100$ cohort and thus demonstrating a reduced intraocular inflammation in patients with a deep AC morphology (Table 2).

In addition, the $\mathrm{AC}$ depth, $\mathrm{AC}$ volume, and $\mathrm{AC}$ angle were significantly larger in the flare $<100$ group compared to the flare 100-249 cohort $10 \mathrm{~min}$ post femtosecond laser treatment $(P=0.002, P=0.023$, $P=0.007)$. The central and thinnest corneal thickness was significantly increased in the higher flare cohorts (100-249 +>249 photon counts/ms) 10 min post femtosecond laser treatment (Table 2); the increase of the corneal thickness was observed in studies of active AC inflammation due to the amount of inflammation and could be confirmed by our study results. ${ }^{21,22}$

Similar findings were demonstrated in the flare $<100$ cohort compared to the flare cohort $>249$; AC depth, AC volume and $\mathrm{AC}$ angle were significantly deeper in the flare $<100$ cohort $10 \mathrm{~min}$ post femtosecond laser treatment $(P=0.001, P=0.007, P=0.003)$. Our findings regarding the AC depth, volume and angle were confirmed by Otsuki et al. ${ }^{23}$ Indeed, changes in AC depth are the most sensitive indicator of inflammatory activity in patients with anterior uveitis due to ciliary oedema. ${ }^{23}$ Summarizing our findings, a flat $\mathrm{AC}$, low $\mathrm{AC}$ volume and a narrow $\mathrm{AC}$ angle were associated with blood barrier breakdown and thus higher 
intraocular inflammation. This is the first study to verify these characteristics in patients to enable surgeons to classify their patients before surgery.

Considering the meaning of endothelial cells, Kacerovská et al ${ }^{24}$ demonstrated that the first day postoperative showed an average decrease in the number of endothelial cells by $1.1 \%$ in the femtosecond laser group; the conventional phacoemulsification group had a decrease of $3.8 \%$. We found a loss of $0.36 \%$ in the normal cataract cohort at 1 day of follow up. Following manual phacoemulsification, an average of $8.5 \%$ cell loss was reported in the literature 12 months postoperatively. ${ }^{25}$

This study is limited by missing features of the AC morphology that were not included in this study design. Schultz et $a^{26}$ noted a significantly higher level of prostaglandins in the aqueous humour of patients immediately after femtosecond laser treatment. No correlation was noted between age or cataract density and prostaglandin E2 level or between corneal incision, suction time or laser time in the femtosecond laser group and prostaglandin E2 level. ${ }^{26}$ A further limitation of this study was the missing manual phacoemulsification control group. In addition, our study was limited by preoperative axial length differences between the flare $<100(24.0 \pm 1.0 \mathrm{~mm})$ and flare 100-249 cohorts $(23.5 \pm 1.3 \mathrm{~mm}, P=0.030)$, which should be corrected in future study designs.

In conclusion, this study was the first to compare the alterations of the AC characteristics due to femtosecond laser-assisted capsulorhexis and photodisruption related to flare photometry. A flat AC, low AC volume, and narrow $\mathrm{AC}$ angle were parameters associated with higher intraocular inflammation. These criteria could be used for patient selection to reduce or prevent unexpected postoperative intraocular inflammation reaction in FLACS.

\section{Summary}

What was known before

- Studies relating to femtosecond laser-assisted cataract surgery (FLACS) evaluated the phacoemulsification time, the intraocular lens placement, the capsule overlap, the circularity of capsulotomy, the centration of the intraocular lens and the central cornea thickness, but not the impact on different anterior segment parameters due to the laser energy.

- Reduction of ultrasound energy in FLACS compared with manual procedure.

\section{What this study adds}

- Flat anterior chamber, low anterior chamber volume and narrow anterior chamber angle are parameters associated with higher intraocular inflammation.

- Criteria can be used for patient selection to reduce or prevent unexpected postoperative intraocular inflammation reaction in FLACS.

\section{Conflict of interest}

The authors declare no conflict of interest.

\section{References}

1 Nagy Z. New technology update: femtosecond laser in cataract surgery. Clin Ophthalmol 2014; 8: 1157-1167.

2 Nagy Z, Mastropasqua L, Knorz M. The use of femtosecond lasers in cataract surgery: review of the published results with the Lensx system. J Refract Surg 2014; 30(11): 730-740.

3 Nagy Z, Takacs A, Filkorn T, Sarayba M. Initial clinical evaluation of an intraocular femtosecond laser in cataract surgery. J Refract Surg 2009; 25: 1053-1060.

4 Kranitz K, Takacs A, Muhaltz K, Kovács I, Knorz MC, Nagy $\mathrm{ZZ}$. Femtosecond laser and manual continuous curvilinear capsulorrhexis parameters and their effects on intraocular lens centration. J Refract Surg 2011; 27: 558-563.

5 Alio JL, Abdou AA, Soria F, Javaloy J, Fernández-Buenaga R, Nagy ZZ et al. Femtosecond laser cataract incision morphology and corneal higher order aberration analysis. J Refract Surg 2013; 29: 590-595.

6 Kohnen T, Klaproth O, Ostovic M, Hengerer F, Mayer W. Morphological changes in the edge structures following femtosecond laser capsulotomy with varied patient interfaces and different energy settings. Graefes Arch Clin Exp Ophthalmol 2014; 252(2): 293-298.

7 Tyndall J. On the blue of the sky, the polarization of the skylight, and on the polarization of light by cloudy matter generally. Philos Mag J Sci 37: 384-404.

8 Hogan MJ, Kimura SJ, Thygeson P. Signs and symptoms of Uveitis. I. Anterior uveitis. Am J Ophthalmol. 1959; 47: 155-170.

9 Ladas JG, Wheeler NC, Morhun PJ, Rimmer SO, Holland GN. Laser flare-cell photometry: Methodology and clinical applications. Surv Ophthalmol 2005; 50: 27-47.

10 Konstantopoulou K, Del'Omo R, Morley AM, Karagiannis D, Bunce C, Pavesio C. A comparative study between clinical grading of anterior chamber flare and flare reading using the Kowa laser flare meter. Int Ophthalmol 2012; 35(5): 629-633

11 Shah SM, Spalton DJ, Taylor JC. Correlations between laser flare measurements and anterior chamber protein concentrations. Invest Ophthalmol Vis Sci. 1992; 33: 2878-2884.

12 Magalhaes FP, Costa EF, Cariello AJ, Rodrigues EB, HoflingLima AL. Comparative analysis of the nuclear lens opalescence by the Lens Opacities Classification System III with nuclear density values provided by Oculus Pentacam: A cross-section study using Pentacam nucleus staging software. Arq Bras Oftalmol 2011; 74: 110-113.

13 Kovacs I, Kranitz K, sandor GL, Knorz MC, Donnenfeld ED, Nuijts RM et al. The effect of femtosecond laser capsulotomy on the development of posterior capsule opacification. J Refract Surg 2014; 30: 154-158.

14 Bernasconi O, Papadia M, Herbort CP. Sensitivity of laser flare photometry compared to slit-lamp cell evaluation in monitoring anterior chamber inflammation in uveitis. Int Ophthalmol. 2010; 30: 495-500.

15 Ursell PG, Spalton DJ, Tilling K. Relation between postoperative blood-aqueous barrier damage and LOCS III cataract gradings following routine phacoemulsification surgery. Br J Ophthalmol. 1997; 81(7): 544-547. 
16 Oshika T, Kato S. Changes in aqueous flare and cells after mydriasis. Jpn J Ophthalmol 1989; 33: 271-278.

17 Liu Y, Luo L, He M, Liu X. Disorders of the blood-aqueous barrier after phacoemulsification in diabetic patients. Eye (Lond) 2004; 18(9): 900-904.

18 Zarnowski T, Chmiel M, Haszcz D, Zagórski Z. Evaluation of astigmatism and blood-aqueous barrier breakdown following procedures in the anterior eye segment. Klin Oczna 1998; 100(1): 11-14.

19 Abell RG, Kerr NM, Vote BJ. Anterior chamber flare after femtosecond laser-assisted cataract surgery. J Cataract Refract Surg. 2013; 39: 1321-1326.

20 Schauersberger J, Kruger A, Müllner-Eidenböck A, Petternel V, Abela C, Svolba G et al. Long-term disorders of the blood-aqueous barrier after small-incision cataract surgery. Eye (Lond) 2000; 14: 61-63.

21 Agra C, Agra L, Dantas J, Faria e Arantes TE, de Andrade Neto JL. Anterior segment optical coherence tomography in acute anterior uveitis. Arq Bras Oftalmol 2014; 77(1): 1-3.

22 Heinz C, Taneri S, Roesel M, Heiligenhaus A. Influence of corneal thickness changes during active uveitis on
Goldmann applanation and dynamic contour tonometry. Ophthalmic Res 2012; 48(1): 38-42.

23 Otsuki T, Shimizu K, Igarashi A, Kamiya K. Usefulness of anterior chamber depth measurement for efficacy assessment of steroid pulse therapy in patients with VogtKoyanagi-Harada disease. Jpn J Ophthalmol 2010; 54(5): 396-400.

24 Kacerovská J, Kacerovský M, Kadlec R. Development of number of endothelial cells after cataract surgery performed by femtolaser in comparison to conventional phacoemulsification. Cesk Slov Oftalmol 2013; 69(5): 215-218.

25 Takacs AI, Kovacs I, Mihaltz K, Filkorn T, Knorz MC, Nagy ZZ. Central corneal volume and endothelial cell count following femtosecond laser-assisted refractive cataract surgery compared to conventional phacoemulsification. J Refract Surg 2012; 28: 387-391.

26 Schultz T, Joachim SC, Kuehn M, Dick HB. Changes in prostaglandin levels in patients undergoing femtosecond laser-assisted cataract surgery. J Refract Surg 2013; 29(11): 742-747. 